\title{
Multiple lymphomatous polyposis: Characteristic endoscopic features
}

\author{
Shah Aiman • Anupam Chakrapani • \\ Satyakam Sawaimoon - Saugata Sen • \\ Mammen Chandy $\cdot$ Suvadip Chatterjee
}

Published online: 10 September 2014

(C) Indian Society of Gastroenterology 2014

Gastrointestinal mantle cell lymphoma (MCL) is an aggressive subtype of B-cell lymphoma accounting for $1 \%$ to $2 \%$ of non-Hodgkin lymphomas. Patients usually present with lymphadenopathy, bone marrow, and extranodal involvement. The commonest gastrointestinal (GI) manifestation is multiple lymphomatous polyposis, in which multiple lymphoid polyps are present in the GI tract $[1,2]$. The ileocecal region is the commonest site involved. GI symptoms include pain, obstruction, diarrhea, or hematochezia. Multiple lymphomatous polyposis may also be seen in marginal B cell lymphomas, mucosa associated lymphoid tissue (MALT), and follicular lymphomas. Despite prompt remission, prognosis remains poor in view of increased relapse rates. Median survival is 3 to 5 years. Our patient presented with bloody diarrhea for 6 weeks. Colonoscopy revealed diffuse submucosal polypoidal lesions (Fig. 1, Supplemental Fig. S1) and upper GI endoscopy revealed similar multiple polypoidal lesions in the stomach (Supplemental Fig. S2) and duodenum.

Electronic supplementary material The online version of this article (doi:10.1007/s12664-014-0497-4) contains supplementary material, which is available to authorized users.

\section{S. Aiman $\cdot$ S. Chatterjee $(\square)$}

Department of Gastroenterology, Tata Medical Center, Kolkata 700156 , India

e-mail: suvadip_chatterjee@yahoo.com

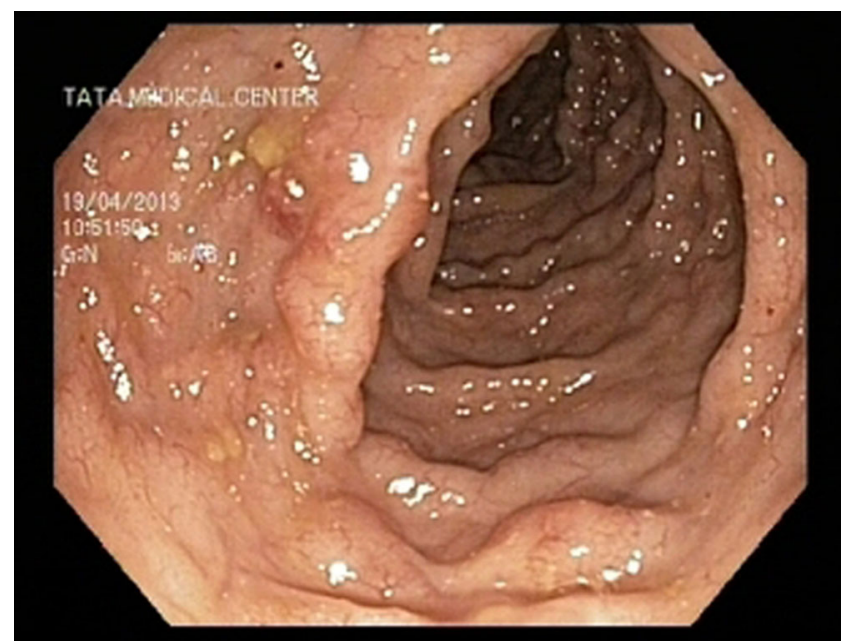

Fig. 1 Pan-colonic lymphomatous polyposis noted on colonoscopy

CT scan abdomen (Supplemental Fig. S3a, b) revealed nodular lesions involving the entire GI tract with intraabdominal lymphadenopathy. Biopsies from the nodules revealed malignant B-cell lymphoma with immunohistochemistry positive for CD20, CD5, and cyclin D1 (Supplemental Fig. S4.) He received rituximab-based chemotherapy which resulted in significant tumor regression (Supplemental Fig. S5) and prompt symptomatic palliation.

\section{References}

1. Isaacson PG, MacLennan KA, Subbuswamy SG. Multiple lymphomatous polyposis of the gastrointestinal tract. Histopathology. 1984;8: 641-56.

2. Triozzi PL, Borowitz MJ, Gockerman JP. Gastrointestinal involvement and multiple lymphomatous polyposis in mantle zone lymphoma. J Clin Oncol. 1986;4:866-73.
S. Sen

Department of Radiology, Tata Medical Center, Kolkata 700156 , India 